\title{
Differential impact of amino acids on OXPHOS system activity following carbohydrate starvation in Arabidopsis cell suspensions
}

\author{
João Henrique F. Cavalcantia,b, Carla G. S. Quinhones ${ }^{a, b}$, Peter Schertl| ${ }^{b}$, Danielle S. Brito ${ }^{a, b}$, \\ Holger Eubel ${ }^{b}$, Tatjana Hildebrandt ${ }^{b}$, Adriano Nunes-Nesi ${ }^{a}$ (D) , Hans-Peter Braun ${ }^{b}$ (D) \\ and Wagner L. Araújo a,* \\ a Max-Planck Partner Group at the Departamento de Biologia Vegetal, Universidade Federal de Viçosa, 36570-900, Viçosa, Minas Gerais, Brazil

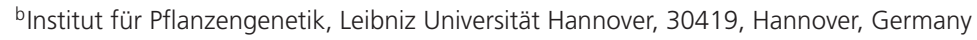

\section{Correspondence \\ ${ }^{*}$ Corresponding author, \\ e-mail: wlaraujo@ufv.br}

Received 10 May 2017;

revised 20 July 2017

doi:10.1111/ppl.12612
Plant respiration mostly depends on the activity of glycolysis and the oxidation of organic acids in the tricarboxylic acid cycle to synthesize ATP. However, during stress situations plant cells also use amino acids as alternative substrates to donate electrons through the electron-transfer flavoprotein (ETF)/ETF:ubiquinone oxidoreductase (ETF/ETFQO) complex to the mitochondrial electron transport chain (mETC). Given this, we investigated changes of the oxidative phosphorylation (OXPHOS) system in Arabidopsis thaliana cell culture under carbohydrate starvation supplied with a range of amino acids. Induction of isovaleryl-CoA dehydrogenase (IVDH) activity was observed under carbohydrate starvation which was associated with increased amounts of IVDH protein detected by immunoblotting. Furthermore, activities of the protein complexes of the mETC were reduced under carbohydrate starvation. We also observed that OXPHOS system activity behavior is differently affected by different amino acids and that proteins associated with amino acids catabolism are upregulated in cells following carbohydrate starvation. Collectively, our results support the contention that ETF/ETFQO is an essential pathway to donate electrons to the $\mathrm{mETC}$ and that amino acids are alternative substrates to maintain respiration under carbohydrate starvation.

\section{Introduction}

The primary role of the mitochondrion in the plant cell, similarly in all eukaryotic cells, is to synthesize ATP through cellular respiration (Millar et al. 2005). Also the mitochondrion plays further roles in plant metabolism such as the production of reducing equivalents and metabolic intermediates for use in biosynthetic pathways elsewhere within the cell (Araújo et al. 2012, Huang et al. 2013). The importance of mitochondrial function in

Abbreviations - ABA, Abscisic Acid; AOX, alternative oxidase; BCAA, branched chain amino acids; D2HGDH, (D)-2-hydroxyglutarate dehydrogenase; DCIP, dichlorophenolindophenol; ETF, electron transfer flavoprotein; ETFQO, ETF:ubiquinone oxidoreductase; GABA, gamma-Aminobutyric Acid; IVDH, isovaleryl-CoA dehydrogenase; KCN, potassium cyanide; LKR/SDH, lysine-ketoglutarate reductase/sacharopine dehydrogenase; mETC, mitochondrial electron transport chain; MS, Murashige and Skoog; MS/MS, tandem mass spectrometry; OXPHOS, oxidative phosphorylation system; PTM, post-translational modification; ROS, reactive oxygen specie; SDS-PAGE, Sodium Dodecyl Sulphate Polyacrylamide gel electrophoresis; SHAM, salicylhydroxamic acid; Suc, sucrose; TCA cycle, tricarboxylic acid cycle.

¥These authors contributed equally to this work. 
the physiology and development of higher plants is also demonstrated by the fact that mutations in several genes of the mitochondrial genome frequently lead to cytoplasmic male sterility (Chase 2007) and by the association of plant mitochondria with programmed cell death (Yao et al. 2004, Reape et al. 2008). In fact, plant respiratory metabolism exhibits several unique features including multiple entry points from sucrose to starch, complementation between cytosol, plastid and mitochondria, and the presence of non-phosphorylating transport systems and alternative pathways of respiration (Fernie et al. 2004, Klodmann et al. 2011, Millar et al. 2011, Schertl and Braun 2014).

The main components of plant respiration, including enzymes of the tricarboxylic acid (TCA) cycle and the protein constituents of the mitochondrial electron transport chain (mETC), have been identified and characterized in several plant species (Millar et al. 2011, Araújo et al. 2012, Cavalcanti et al. 2014). However, our understanding of the regulation of the responses of both classical and alternative pathways of plant respiration in response to environmental conditions is still limited. Although it was demonstrated that the oxidation of alternative substrates is of high importance during several stress situations that affect carbohydrate supply (Araújo et al. 2010, Araújo et al. 2011, Engqvist et al. 2011), plant respiration is mostly dependent on carbohydrate oxidation (Paxton and Podesta 2006). However, under some circumstances, such as natural senescence or environmental stresses, carbohydrate levels are reduced forcing the plant cells to use alternative substrates to support ATP synthesis (Araújo et al. 2011). In this scenario, protein degradation can be an important source of alternative substrates for maintaining mitochondrial energy metabolism under suboptimal conditions (Ishizaki et al. 2005, Araújo et al. 2011).

It seems clear that some features of respiration are distinct from those of the classical respiration when alternative substrates are used. For instance, pathways linking branched-chain amino acids (BCAA) and lysine degradation to electron donation to the mETC through the action of alternative dehydrogenases have been identified. By the action of the alternative dehydrogenases, electrons are donated directly to the electron-transfer flavoprotein (ETF)/ETF:ubiquinone oxidoreductase (ETF/ETFQO) complex and are afterwards transferred to the ubiquinone pool bypassing complexes I and II (Araújo et al. 2010, Kleessen et al. 2012). In mammals, the presence of 11 dehydrogenases able to donate electrons to this alternative pathway has been demonstrated (Beckmann and Frerman 1985). In plants, however, only two major dehydrogenases - isovaleryl-CoA dehydrogenase (IVDH) and (D)-2-hydroxyglutarate dehydrogenase
(D2HGDH) - that oxidize BCAA (isoleucine, leucine and valine) and lysine, respectively, have been characterized to date (Enqvist et al. 2009, Araújo et al. 2010). By using a combination of genetic, biochemical and molecular approaches ethylmalonic encephalopathy protein 1 (ETHE1), which is involved in cysteine catabolism, has been identified as another enzyme associated with the ETF/ETFQO system (Krüßel et al. 2014). Moreover, proline dehydrogenase, which is located in the inner mitochondrial membrane, has been shown to donate electrons to the mETC as well (Schertl et al. 2014). Collectively, these findings suggest that the ETF/ETFQO complex, similarly to the situation observed in mammals, can be regarded as a branching point of the mETC.

In plants, degradation of amino acids for maintenance of respiration has been shown to be important during carbon deprivation (Araújo et al. 2010) and metabolite analysis of the mitochondrial composition following oxidative stress suggested that by increasing amino acid catabolism plants might compensate for a reduced electron supply from the TCA cycle (Obata et al. 2011). Notably, not only ETF/ETFQO enzymes but also other alternative enzymes, mainly IVDH, have been demonstrated to be upregulated under a range of environmental stresses (Zhou et al. 2003, Karvchik and Bernstein 2013). To better understand the role of alternative substrates for electron supply to the mETC under carbohydrate starvation we performed a range of experiments using Arabidopsis cell culture following carbohydrate starvation for $24 \mathrm{~h}$ and supplied with different amino acids as alternative substrates. We focused our analysis on $24 \mathrm{~h}$ only because it was previously demonstrated that shorter time of carbohydrate starvation usually lead to minor changes in total respiration, sugars content and cytoplasmic volume (Journet et al. 1986, Roby et al. 1987) whereas major morphological, metabolic and transcriptional changes are observed following longer periods of carbohydrate starvation (Contento et al. 2004, Nicolai et al. 2006, Kim et al. 2007). We also decided to work with BCAA and lysine because they represent alternative electron donors at the mitochondrial level. Our results demonstrate an opposite behavior among components of OXPHOS system and provide evidence that changes in amino acid sources drive metabolic adaptation of Arabidopsis cells, especially regarding the mETC. Furthermore, by using a label free liquid chromatography coupled tandem mass spectrometry (LC-MS/MS) proteomics approach we demonstrate the occurrence of a general mitochondrial reprogramming which applies to all conditions used here but also highlights the presence of specific changes in the mitochondrial proteome following 


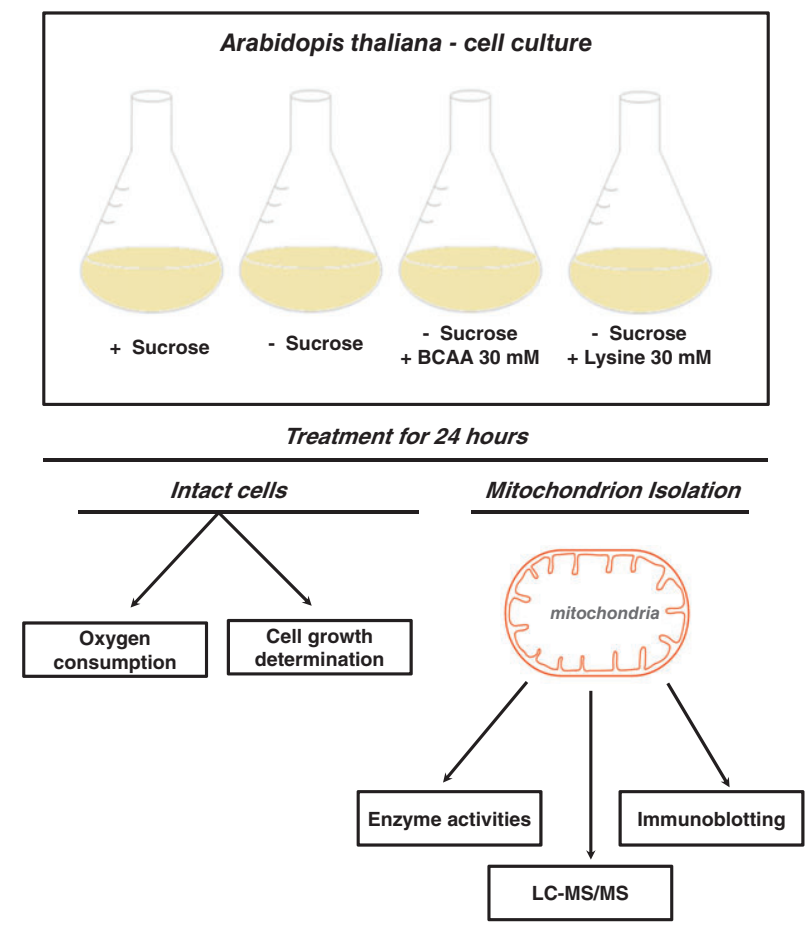

Fig. 1. Experimental strategy for investigating effects of carbohydrate limitation in Arabidopsis cells. Cell suspension were cultivated under the following conditions: (1) +sucrose ( $3 \%$ sucrose; control conditions), (2) no sucrose, (3) BCAA [without sucrose plus $10 \mathrm{mM}$ BCAA (10 $\mathrm{mM}$ isoleucine, $10 \mathrm{mM}$ leucine and $10 \mathrm{mM}$ valine)] and (4) lysine (without sucrose plus $30 \mathrm{mM}$ lysine). After cultivation for $24 \mathrm{~h}$ in the conditions described above further analysis were performed as shown.

carbohydrate starvation plus supplementation with BCAA or lysine.

\section{Results}

\section{The effects of carbohydrate starvation on cell growth and cell respiration}

To estimate the impact of carbohydrate limitation on cell cultures an experimental setup was established as described in Fig. 1. Briefly, cell cultivation was performed in four conditions: (1) control (3\% sucrose), (2) no sucrose, (3) BCAA (without sucrose plus $30 \mathrm{mM}$ BCAA $(10 \mathrm{mM}$ isoleucine, $10 \mathrm{mM}$ leucine and $10 \mathrm{mM}$ valine) and (4) lysine (without sucrose plus $30 \mathrm{mM}$ lysine). After cultivation for $24 \mathrm{~h}$ in the conditions described above we analyzed cell viability and growth. Cell viability of cells under all conditions tested was higher than $70 \%$ (data no shown). Although not statistically significant under carbohydrate starvation conditions (absence of sucrose), a reduction in the cell growth was observed independent of the amino acid supplied (Fig. 2). The presence of amino acids (BCAA or lysine) did not promote growth

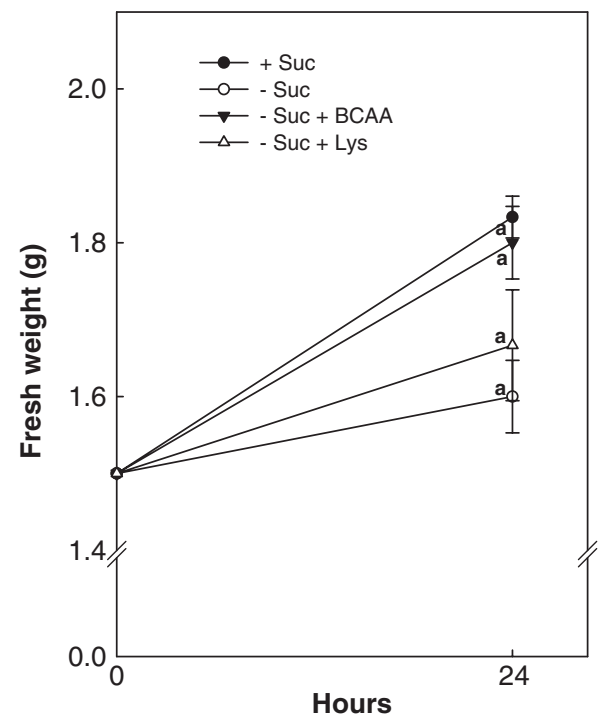

Fig. 2. Cell growth under carbohydrate starvation conditions in Arabidopsis thaliana Col-0 cell culture. Analyses are based on the weight of cells of $A$. thaliana Col-0 after cultivation for 0 and $24 \mathrm{~h}$ at the following conditions: (1) with sucrose; (2) without sucrose; (3) without sucrose plus $30 \mathrm{mM}$ BCAA (10 $\mathrm{mM}$ of each: valine, isoleucine and leucine); (4) without sucrose plus $30 \mathrm{mM}$ lysine. Small letters: student Newman Keuls $(P<0.05)$ significant difference between conditions (carbohydrate starvation) after $24 \mathrm{~h}$ of treatment.

when compared with cells growing in absence of sucrose for $24 \mathrm{~h}$.

Next we investigated the impact of carbohydrate starvation on respiration and the importance of amino acids as alternative substrates to the mETC. To this end, oxygen consumption measurements were carried out in cells submitted to carbohydrate starvation conditions for $24 \mathrm{~h}$ (Fig. 3). Additionally, the effects of the mETC inhibitors $\mathrm{KCN}$ and SHAM were analyzed. As expected, carbohydrate starvation conditions reduced respiratory rates by about $30 \%$ in all conditions (Fig. $3 \mathrm{~A}$ ). In addition, the addition of KCN, an inhibitor of complex IV, decreased respiration rates by around $50 \%$ either in the presence or in the absence of sucrose (Fig. 3B). Interestingly, in the presence of BCAAs the respiration rate was reduced by $\mathrm{KCN}$ by only $30 \%$, and $\mathrm{KCN}$-application did not inhibit the respiratory rates in the presence of lysine at all, indicating a high degree of $\mathrm{KCN}$-resistant respiration. However, we also observed higher relative oxygen consumption rates following the addition of KCN plus SHAM in conditions with absence of sucrose plus lysine followed by the presence of BCAAs (Fig. 3B), most likely associated with stimulation of the cyanide-sensitive oxidase (de Visser and Blacquiere 1984) or peroxidases (Brouwer et al. 1986) by SHAM. Notably, the highest relative reduction was observed in presence of sucrose (our control condition - Fig. 3B). 


\section{Isovaleryl-CoA dehydrogenase is induced following carbohydrate starvation}

To investigate the behavior of alternative enzymes of respiration following carbohydrate starvation we turned our attention to the activity of IVDH, which was differently affected by our treatments (Fig. 4). As expected, the strongest induction of IVDH activity was observed in absence of sucrose (-Suc) and in presence of BCAA $(-\mathrm{Suc}+\mathrm{BCAA})$ which resulted in increments of 1.8and 2.2-fold in IVDH activity, respectively (Fig. 4A). Cultivation of the cells in the presence of sucrose led to basal IVDH activity. IVDH activity in absence of sucrose but supplied with lysine culminated with similar IVDH levels as cultivation in the presence of sucrose. This is not particularly surprising given that lysine is catabolized by $\mathrm{D} 2 \mathrm{HGDH}$. To investigate whether the changes in IVDH activity were caused by enzyme activation and/or de novo synthesis of IVDH we carried out an immunoblotting experiment. Mitochondrial fractions were separated by SDS/PAGE, transferred to nitrocellulose membranes by electroblotting and probed using an antibody directed against IVDH. An immunopositive band at $42 \mathrm{kDa}$ corresponds nicely to the molecular mass of IVDH. In good agreement with the enzymatic activity assay, IVDH was detected in mitochondrial fractions from all cell cultivation conditions. Highest IVDH levels were observed upon cultivation in -Suc and -Suc + BCAA (Fig. 4B).

\section{OXPHOS system show a different behavior under carbohydrate starvation when supplied with a range of amino acids}

To investigate whether the cultivation of Arabidopsis cells following carbohydrate starvation changes the activity of mETC complexes as well as of dehydrogenases located on the inner mitochondrial membrane we analyzed the maximum activities of complexes I, II, III and IV. Interestingly, carbohydrate starvation caused diverging effects on the activities of the OXPHOS complexes. While activities of complex I, and the alternative $\mathrm{NADH}$ dehydrogenases as well as the activities of the complexes III and IV were negatively affected by -Suc and -Suc + BCAA conditions, but not by -Suc + Lys, the activity of complex II is similar to control conditions (Fig. 5). The activity of complex I and alternative $\mathrm{NADH}$ dehydrogenases was significantly reduced following carbohydrate limitation. It was also observed that the addition of lysine reversed the carbohydrate deficiency effect (Fig. 5A). As the complex I and alternative NADH dehydrogenases use the same substrate, $\mathrm{NADH}$, the activity of complex I was inhibited by the addition of rotenone, allowing distinguishing between the activity of complex I of the ones of the alternative $\mathrm{NADH}$
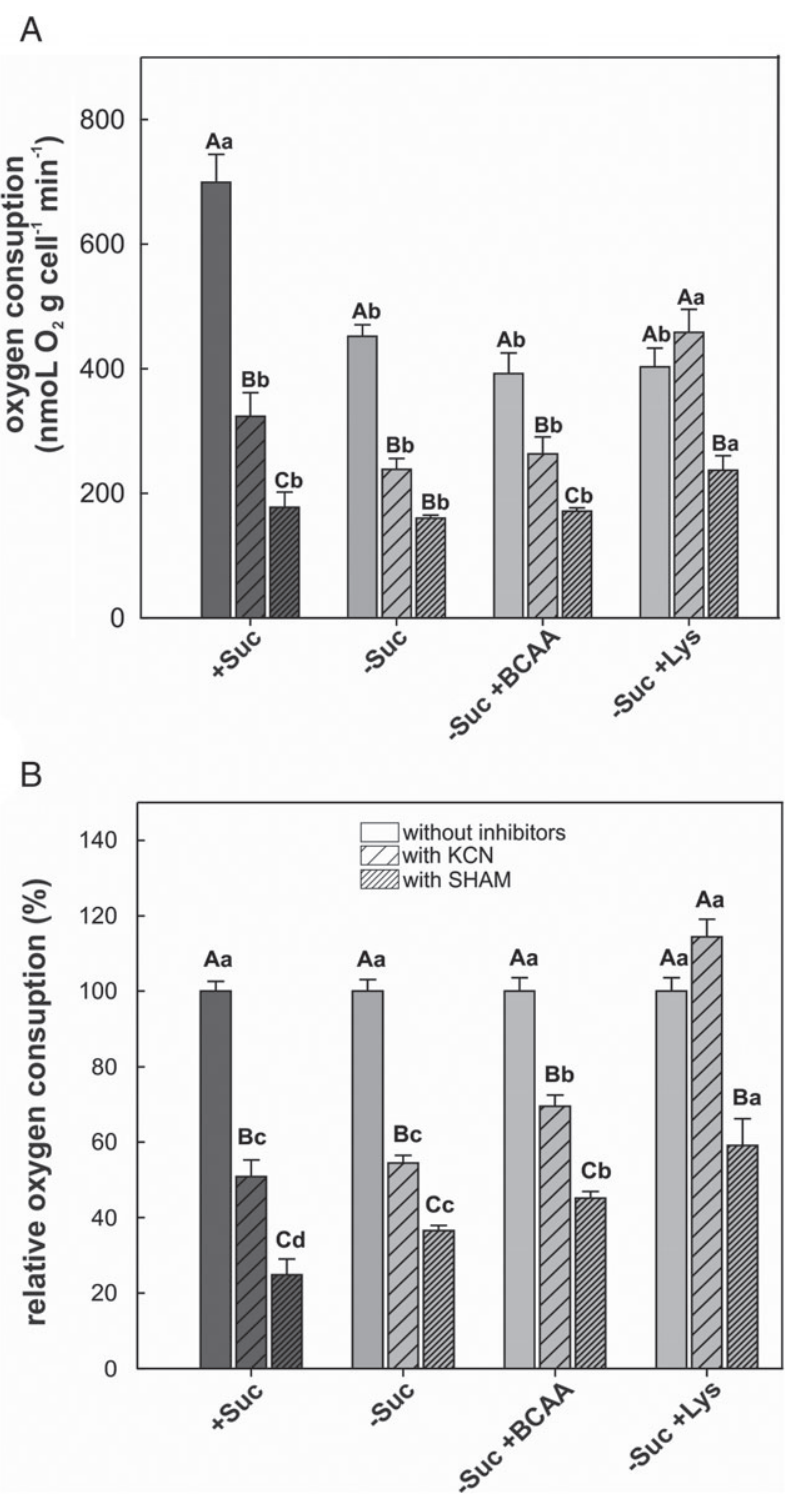

Fig. 3. Oxygen consumption measurements using a Clark-type electrode of Arabidopsis thaliana cells cultivated at different carbohydrate starvation conditions: (1) with sucrose; (2) without sucrose; (3) without sucrose plus $30 \mathrm{mM}$ BCAA (10 $\mathrm{mM}$ of each: valine, isoleucine and leucine); (4) without sucrose plus $30 \mathrm{mM}$ lysine. Inhibitors of cytochrome c oxidase [potassium cyanide (KCN) and the alternative oxidase salicylhydroxamic acid (SHAM)] were added at defined time points (see section Material and methods). (A) Absolute oxygen consumption, (B) relative oxygen consumption (relative oxygen consumption was determined for all conditions to the oxygen consumption obtained without inhibitors, which is considered as $100 \%$ ). Small letters: student Newman Keuls $(P<0.05)$ significant difference between conditions (carbohydrate starvation) in the presence of the same inhibitor. Capital letters: $t$-test $(P<0.05)$ significant effect of different inhibitors within the same conditions. Standard errors are based on three biological replicates. 
A

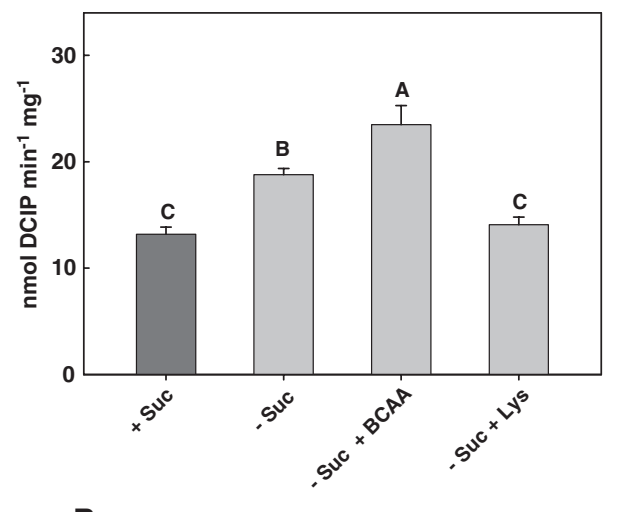

B
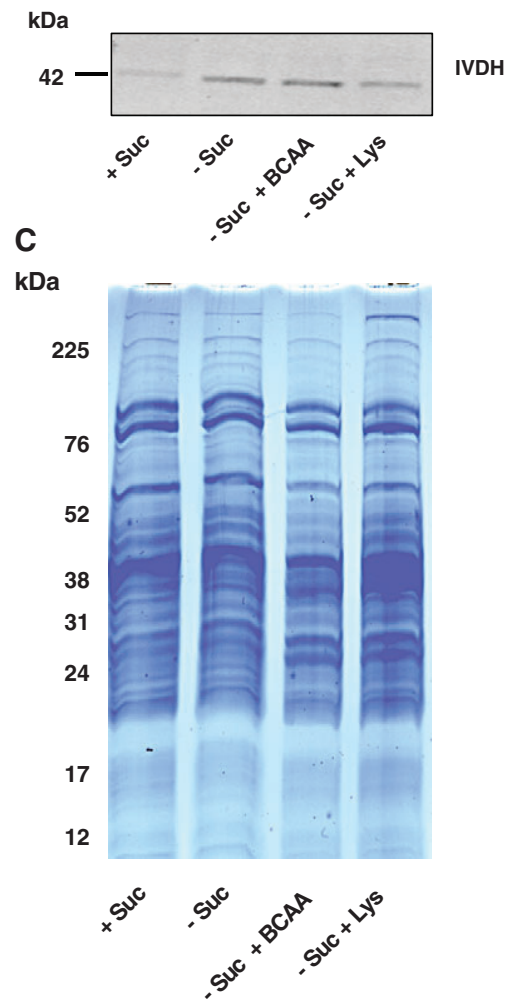

Fig. 4. Induction of isovaleryl-CoA dehydrogenase in Arabidopsis thaliana Col-0 cell suspension following carbohydrate starvation. (A) Activity of isovalery-CoA dehydrogenase (IVDH) was measured in mitochondria isolated from $A$. thaliana cells. Values are means of three biological repetitions and different letters indicate values that were determined by the Student's $t$-test to be significantly different $(P<0.05)$. (B) Identification of IVDH by immunoblotting. Mitochondrial proteins isolated from A. thaliana cells were analyzed by immunoblotting using IVDH antibodies. The molecular mass of a standard protein is indicated to the left of the gel. (C) SDS-PAGE of mitochondrial soluble fraction. All analyses were performed using soluble fractions. Order of cultivation conditions in (A), (B) and (C) was the following (from left to right): (1) plus sucrose (control); (2) without sucrose; (3) without sucrose plus $30 \mathrm{mM}$ BCAA (10 mM of each: valine, isoleucine and leucine) and (4) without sucrose plus $30 \mathrm{mM}$ lysine. dehydrogenase (Fig. 5B). It was observed that the overall reduction of $\mathrm{NADH}$ oxidation is most likely associated with decreased complex I activity (Fig. 5B). In addition, it was found that this reduction can be reversed by the addition of lysine or BCAA (Fig. 5B). The activity of complex II (succinate:ubiquinone oxidoreductase) was similar under all conditions tested here following $24 \mathrm{~h}$ carbohydrate starvation (Fig. 5C). It was also found that the activity of the complex III under carbohydrate limitation conditions was reduced in absence of sucrose alone or with the BCAA supplementation (Fig. 5D). However, it remained unchanged in the presence of lysine (Fig. 5D). Regarding the activity of complex IV (cytochrome c oxidase) the strongest reduction was observed under carbohydrate starvation conditions (no sucrose - Fig. 5E). Minor reductions in the activity of complex IV were observed following carbohydrate starvation and supplementation with BCAA (Fig. 5E) whereas an increased activity (in comparison to control conditions) was observed in cell cultivated without sucrose but supplemented with lysine (Fig. 5E), thereby demonstrating a role for alternative pathways that use this amino acid following carbohydrate limiting conditions.

Given the changes observed in the activity of the complexes of the mETC (Fig. 5) we investigated whether it was correlated with protein amount. To this end, immunoblots were carried out to quantify OXPHOS complexes by using specific antibodies against some subunits of the respiratory complexes (Peters et al. 2012). No changes of the amounts of the subunits tested here (complex I-V) were observed (Fig. 6), indicating that the changes observed in enzyme activity (Fig. 5) were most likely associated with enzymatic regulation on another level than protein abundance.

\section{Carbohydrate starvation modifies the mitochondrial proteome}

In order to investigate the changes in the mitochondrial proteomes following carbohydrate starvation, we performed a label-free quantitative liquid chromatography tandem-mass spectrometry (LC-MS/MS, 'shotgun') analysis of three independent mitochondria isolations from each treatment. This approach allowed us to identify 1417 proteins across all four conditions (Table S1, Supporting Information). We found 69, 115 and 62 upregulated proteins whereas 162, 265 and 225 downregulated proteins $(P<0.05)$ in - Suc, - Suc + BCAA and - Suc + lysine treatment, respectively (Fig. 7A). For a comprehensive overview regarding the major changes in mitochondrial metabolism a PageMan> analysis (Usadel et al. 2006) was performed, which gives an overview of functional categories from our dataset, 
A

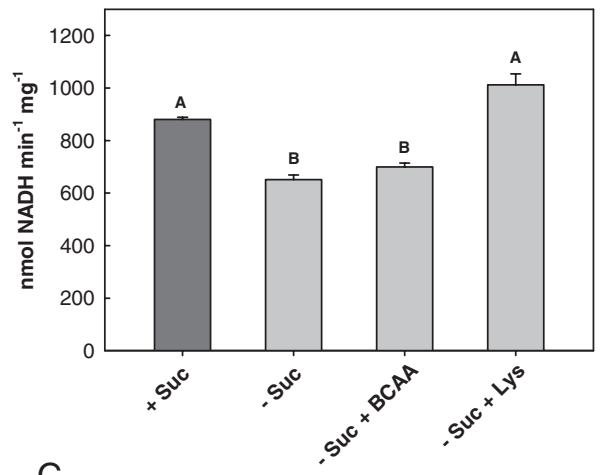

$\mathrm{C}$

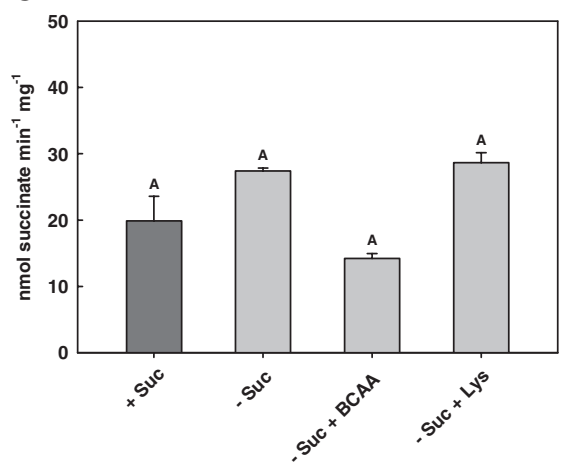

B
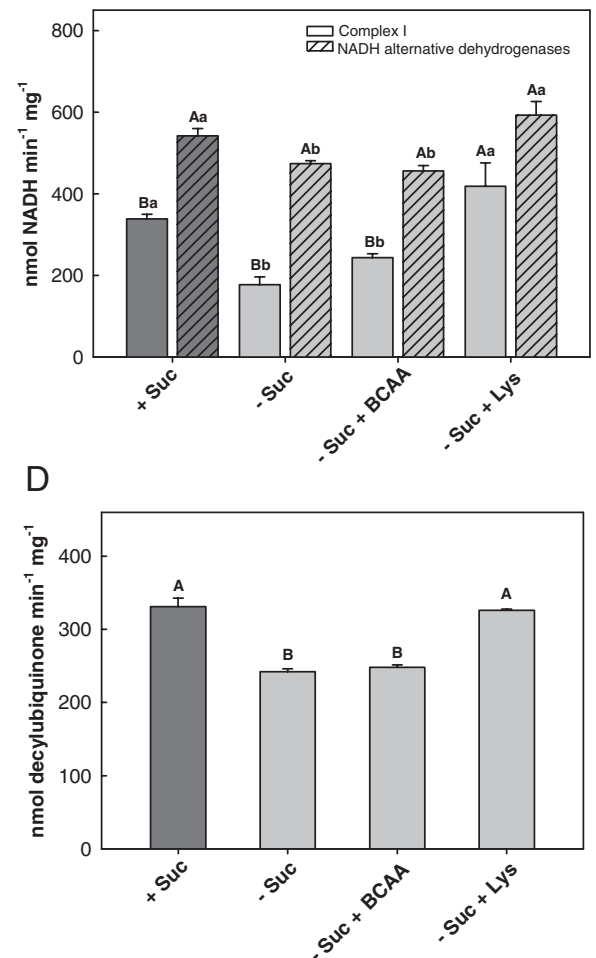

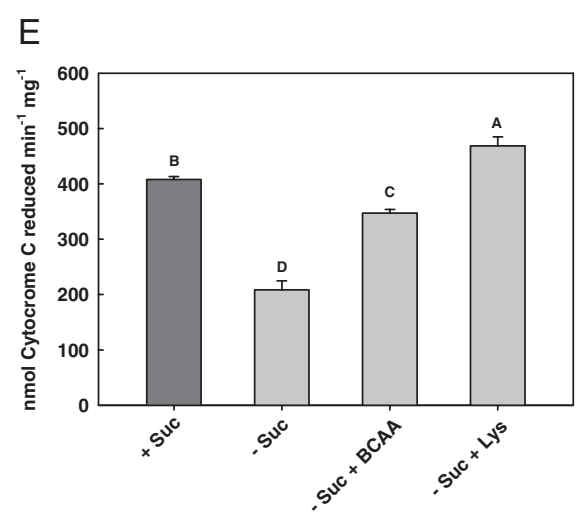

Fig. 5. Activities of complexes I-IV and the alternative NADH dehydrogenases upon cultivation of Arabidopsis cell suspensions for $24 \mathrm{~h}$ at different carbohydrate starvation conditions as measured by photometric enzyme activity assays. (A) Activity of complex I and alternative NADH dehydrogenases. (B) Activity of complex I or alternative NADH dehydrogenases. (C) Activity of complex II. (D) Activity of complex III. (E) Activity of complex IV. OXPHOS system activities were determined using membrane fractions to allow protein enrichment. Columns represent averages of three biological replicates (mitochondrial isolations obtained independently). Different capital letters represent values that were judged to be statistically different $(P<0.05)$ between conditions (carbohydrate starvation). In (B), small letters indicate values that were significant $(P<0.05)$ different between conditions.

using the Wilcoxon test (Fig. S1). The functional categories related to degradative pathways were generally strongly induced (e.g. sucrose degradation, fatty acid degradation by ß-oxidation, GABA synthesis and amino acid degradation group). Interestingly, gluconeogenesis was also induced under carbon starvation. On the other hand, biosynthetic pathways (e.g. amino acid synthesis, nucleotide and protein synthesis) showed to be repressed in our experimental condition. Furthermore, most of the downregulated proteins are members of functional classes like aspartate-family synthesis and protein folding as well as cell cycle and oxidative pentose phosphate pathway.

Because our interest is associated to the mitochondrial energy metabolism, we focused our protein profiling by analyzing the TCA cycle enzymes and alternative dehydrogenase that work as electron donor to the mETC as well as enzymes involved in the oxidative 
A

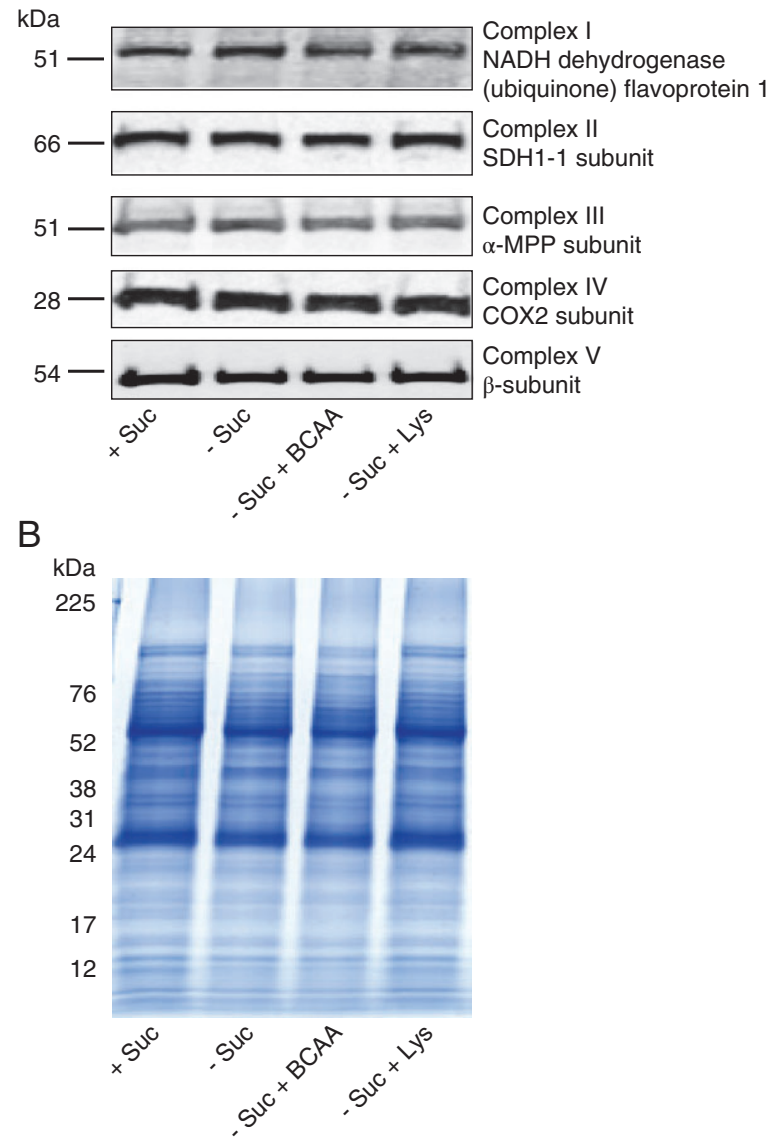

Fig. 6. Immunological quantification of OXPHOS subunits of Arabidopsis thaliana Col-0 cell suspension following carbohydrate starvation. (A) Immunological quantification of subunits of complexes I-V upon cultivation of $A$. thaliana Col-0 cell suspensions at different carbohydrate starvation conditions. (B) Mitochondrial membrane proteins were separated by SDS-PAGE. Immunological quantification of OXPHOS subunits were determined using membrane fractions. (A) and (B) was the following (from left to right): (1) plus sucrose (control); (2) without sucrose; (3) without sucrose plus with $30 \mathrm{mM}$ BCAA (10 mM of each: valine, isoleucine and leucine) and (4) without sucrose plus with $30 \mathrm{mM}$ lysine.

phosphorylation. Twenty-one proteins are upregulated in all conditions (Fig. 7A). Notably, this cluster contains a core set of proteins related to amino acid degradation including IVDH (At3g45300), Enoyl-CoA dehydrogenase (At4g29010), Acyl-CoA dehydrogenase (At5g65110) and ETFQO (At2g43400). Furthermore, we also found inner membrane proteins and/or respiratory subunits located in this cluster including alternative oxidase $1 \mathrm{a}$ (At3g22370), NAD(P)H dehydrogenase B2 (At4g05020), cytochrome BC1 synthase (At3g50930), ATP synthase subunits G (At4g29480) and B (AtMg00640) and proline dehydrogenase 1 (At3g30775). Proteins differently expressed in a unique treatment or in common cluster between two treatments were also observed. Those proteins found in our label free quantitative LC-MS/MS proteome analysis are further described in Tables S2 and S3. Briefly, carbohydrate starved cells with BCAA supplementation displayed the higher number of upregulated proteins. As expected, most of them are related to the catabolism of BCAA but mitochondrial carriers and TCA enzymes isoforms were also found. Remarkably, some of the TCA proteins were identified as peroxisome isoforms most likely because of highly conserved amino acids sequence among TCA cycle isoforms. Moreover, proteins that were upregulated only in cells cultivated in the absence of sucrose included only few dehydrogenases, namely alanine: glyoxylate aminotransferase (At4g39660 and At2g38400) and uncoupling protein 5 (UCP5 - At2g22500). These proteins are recognized to link amino acid or organic acid catabolism to energy metabolism (Hildebrandt et al. 2015). Lysine treatment promoted an induction of isocitrate dehydrogenase 5 (At5g03290) as well as enzymes related to one-carbon metabolism: formate dehydrogenase and (At5g14780) and methionine adenosyltransferase 3 (At2g36880). Moreover, we observed that several proteins were clustered in two treatments. Thus, common proteins upregulated following carbohydrate starvation (-Suc and -Suc + BCAA) included proteins involved in energy metabolism and amino acid catabolism. We also found that enzymes associated with the GABA shunt including the GABA transaminase (At3g22200) were upregulated in cells treated with -Suc + Lys and -Suc + BCAA whereas the glutamate dehydrogenase 2 (At5g07440) was upregulated in all treatments. Similar results were also observed in our PageMan analysis which shows that amino acids degradation and classical mitochondrial electron transport are usually downregulated (Fig. 7B).

\section{Discussion}

During the last decade, we have witnessed an increase in our knowledge concerning the key function of amino acid sensing and the importance it has on virtually every aspect of plant metabolism (Galili 2011, Kirma et al. 2012, Galili et al. 2014). This fact notwithstanding, our current understanding of the connections between protein degradation and amino acid catabolism during carbon starvation (here called carbohydrate limitation) in feeding electrons into the $\mathrm{mETC}$ remains fragmented. In this context, a suitable approach to increase our knowledge concerning carbohydrate starvation and its effect on mitochondrial metabolism in general is the use of Arabidopsis cell suspension. We used this system because of its biological homogeneity, relatively fast growth coupled with rapid response to changes in growth conditions associated with the facility of 
A

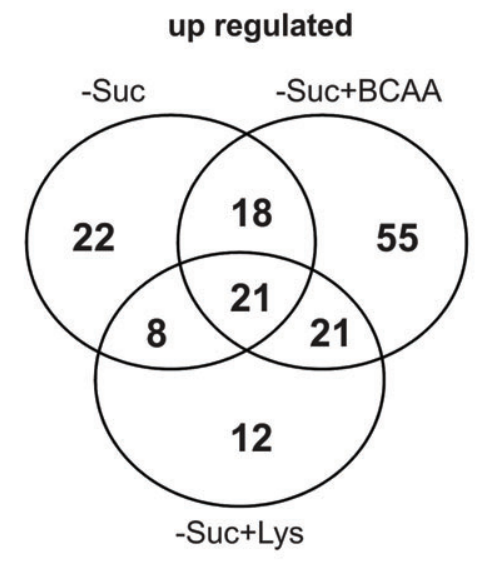

down regulated

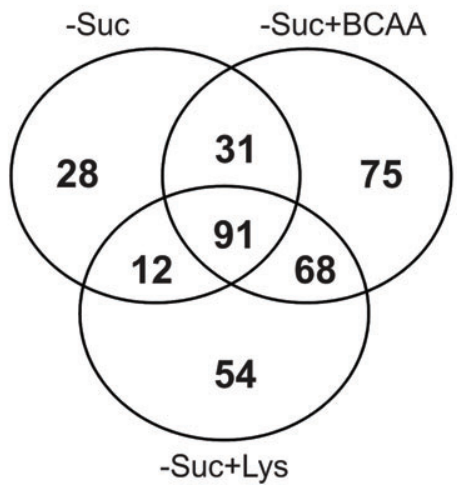

B

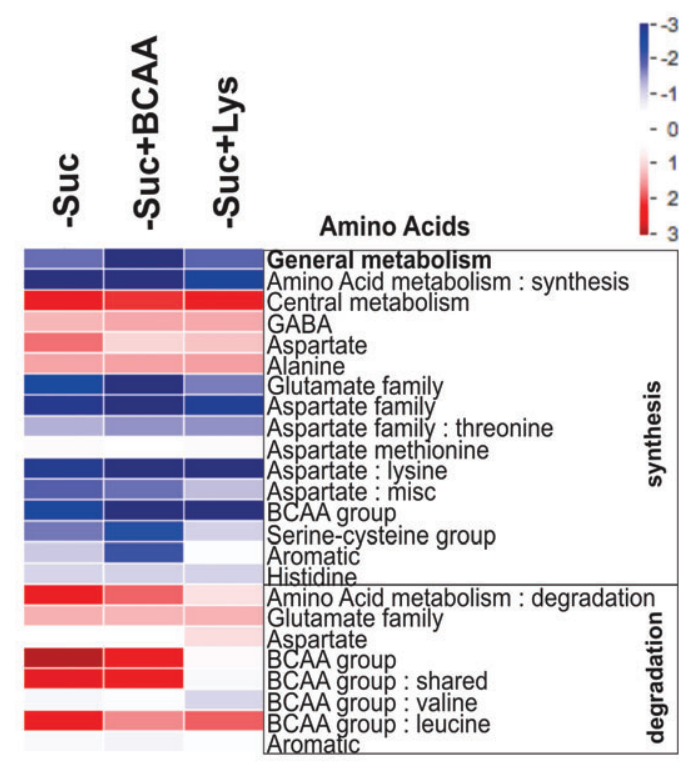

mECT/ATP synthesis

General metabolism

NADH dehydrogenase (Complex

$\mathrm{NADH}$ dehydrogenase Complex

$\mathrm{NADH}$ alternative dehydrogenase

ETFQO

Alternative oxidase (AOX)

Cytochrome $\mathrm{c}$ reductase

Cytochrome c

Cytochrome $\mathrm{c}$ oxidase

Cytochrome c oxidase
Uncoupling protein (UCP)

Fig. 7. Proteomics analysis of Arabidopsis cell suspension after $24 \mathrm{~h}$ under carbohydrate starvation. (A) Venn's diagram comparison among -Suc, -SUC + BCAA and -Suc + Lys treatments. (B) PageMan analysis revealing over-representative functional categorization of differential expressed proteins along carbohydrate starvation. Following mitochondrial isolation, total proteins were used for LC-MS/MS. Red and blue color state up- and down over-represented functional categorization of identified protein, respectively.

mitochondrial isolation, which is of fundamental importance to answer our main questions. This approach has been used in the past to investigate several aspects of carbohydrate starvation including the identification of differentially expressed genes or metabolic changes following starvation (Contento et al. 2004, Nicolai et al. 2006, Kim et al. 2007). It has also been recently used in fluxomic studies to investigate the fluctuation of metabolic networks following physiological perturbations such as the availability of nutrients (Kruger et al. 2012, Masakapalli et al. 2014). However, to our knowledge this is the first attempt to understand changes in the OXPHOS system following carbohydrate starvation and the use of amino acids as alternative substrates to maintain/sustain respiration during energetic stress.

Here, we took advantage of this method by removing sucrose from the media, inducing a carbohydrate starvation, and simultaneously supplying the media with different amino acids. This approach revealed insights into the metabolic mechanisms through which the supply of alternative substrates might promote changes into the mETC. Our results demonstrate that changes in mETC-activities take place following carbohydrate starvation and that those alterations are modified by the amino acid supplied in the medium. Collectively, our results also indicate these changes are apparently associated with the alternative donation of electrons to the ubiquinone pool which is likely mediated by dehydrogenases such as IVDH (Fig. 4). First, by measuring the activity of IVDH we could confirm that our experimental conditions were able to induce changes on mitochondrial energy metabolism (Fig. 4). As could be expected, the control condition containing sucrose displayed the lowest (basal) IVDH activity. In good agreement with previous reports (Araújo et al. 2010, Gu et al. 2010), BCAA were able to promote the highest induction on IVDH activity in the absence of Suc. The highest activation of IVDH in presence of BCAA is in accordance with 
a cumulative effect of both carbohydrate starvation and the addition of the substrate for this enzyme. It should be mentioned, however, that in absence of sucrose we also observed an induction on IVDH activity. It is reasonable to assume that this result is most likely related to protein degradation and recycling of amino acids (Araújo et al. 2011, Hildebrandt et al. 2015) and thus free BCAA released from protein degradation is used for alternative respiration. Although protein degradation might release only a relatively small amount of free amino acids it is possible that this could be enough to induce IVDH activity given that it has a low $\mathrm{Km}$ value of about $1.0 \mu M$ (Weigelt et al. 2008, Mohsen and Vockley 2015). IVDH activity was not altered in presence of lysine even though it has been described as an electron donor to the ETF/ETFQO system (Araújo et al. 2010). Indeed, lysine catabolism has been extensively linked to mitochondrial metabolism and energy production by the action of both lysine-ketoglutarate reductase/sacharopine dehydrogenase (LKR/SDH) or D2HGDH which provide acetyl-CoA and 2-oxoglutarate, respectively (Zhu and Galili 2004, Enquivist et al. 2009, 2011, Galili 2011, Kirma et al. 2012). The action of theses enzymes, which are thought to operate functionally similar albeit independently from the IVDH pathway, will also culminate with the donation of electrons to the ETF/ETFQO system (Kleessen et al. 2012). Given that lysine did not culminate in differences in complex II activity (Fig. 5C) it seems reasonable to assume that both pathways of lysine catabolism act together to support the classical mETC.

Our findings provide additional evidence corroborating the recent suggestion that alternative substrates are used by oxidative pathways of respiration that are induced under carbohydrate starvation (Araújo et al. 2011) and are consistent with the mathematical model built for alternative substrates (e.g. amino acids) used by alternative dehydrogenases for energy generation associated with the ETF/ETFQO system (Kleesen et al. 2012). One conspicuous feature concerning the increased IVDH activity is its correlation with the enhancement of IVDH protein amount as detected by immunoblotting assays and LC-MS/MS data (Fig. 4B; Table S2). Thus, it seems that IVDH activity must be tightly regulated by de novo synthesis and that carbohydrate starvation is able to trigger IVDH transcription. In fact, extended darkness in Arabidopsis, which resulted in decreased carbohydrate levels, was already described to stimulate transcription of IVDH (Buchanan-Wollaston et al. 2005, Ishizaki et al. 2005). Although here we have used Arabidopsis cell suspensions, our results can be compared with mitochondrial metabolic changes in others tissues under stress situation or natural developmental conditions which result in diminished carbohydrate levels. In fact, previous studies using transcriptome analyses of natural or induced senescence leaf tissue showed regulation of genes associated with protein degradation, use of alternative substrates for respiration and induction of genes such as IVDH, ETF and ETFQO (Buchanan-Wollaston et al. 2005). Notably, the work above mentioned has not provided any data concerning the enzymatic activity of OXPHOS system and as such the effective comparison should be taken with caution. Thus, according to our finding it is most likely that during normal leaf senescence OXPHOS system activity occur in a very similar fashion as that observed in cell suspension under sucrose starvation.

To the best of our knowledge, this is the first demonstration of alterations in the activity of the mitochondrial complexes in plants following carbohydrate starvation and perhaps more importantly that during carbohydrate limitations the presence of amino acids, such as lysine and the BCAA, can induce changes in the mitochondrial activity. Although it is well known that carbohydrate starvation triggers alternative respiration (Ishizaki et al. 2005, 2006, Araújo et al. 2010, Krüßel et al. 2014, Peng et al. 2015) our results add novel insights into the regulation of mitochondrial metabolism by showing that amino acids act differently on the mETC.

In this context, it is believed that respiration has a key role in metabolic reprogramming possibly being inhibited under conditions of low availability of carbon as a function of substrate limitation. Moreover, cell growth is a high energy demanding processes that requires an intense metabolic reprogramming. Remarkably, no significant differences on cell growth were observed under the carbohydrate starvation conditions tested here, suggesting that the lack of energy supply might be minimal during the first $24 \mathrm{~h}$ of carbohydrate limitation or perhaps more intriguingly that protein degradation and the use of amino acids can fulfill the energy demand during this period by a reprogramming of metabolic pathways involved in energy production. In good agreement with our decision to analyze cell response following $24 \mathrm{~h}$ of carbohydrate starvation, we did not observe significant changes in both cell growth and cell viability. Thus, the metabolic reprogramming demonstrated by the changes in the OXPHOS system is probably taking place independently of a higher degree of stress, as deduced by the minimal effect on growth and considering that cell viability is not affected yet.

To check the effects of carbohydrate limitation conditions on respiration and the role of amino acid degradation pathways as alternative sources of electrons to the $\mathrm{mETC}$, oxygen consumption measurements were performed in cell cultures. In addition, we evaluated the effects of applying mitochondrial electron chain 
inhibitors, such as KCN and SHAM. As expected, carbohydrate limitation conditions reduced the respiration rate to approximately $70 \%$, approximately under carbohydrate limitation conditions with concomitant use of amino acids for activation of alternative routes. Moreover, the application of $\mathrm{KCN}$ was not sufficient to inhibit the respiration rate in the presence of lysine, suggesting a KCN-resistant respiration. Further metabolic studies should also investigate this intriguing feature. Kühn et al. (2015) showed a 50\% increase in resistant respiration to $\mathrm{KCN}$ in Arabidopsis thaliana plants grown under stress conditions. Also, cell respiration is usually enhanced by the presence of SHAM as reported in both Pisum sativum L. and Plantago lanceolate (de Visser and Blacquiere 1984) and the recognized side effects of SHAM have been previously suggested (de Visser and Blacquiere 1984, Brouwer et al. 1986). It is believed that this fact is a consequence of the increased contribution of the alternative oxidase (AOX) for reducing oxygen (Kühn et al. 2015). In good agreement with these findings, our proteome approach revealed increased levels of proteins associated with alternative respiration that are located at the inner mitochondrial membrane (alternative oxidase, BCS1, alternative $\mathrm{NADH}$ dehydrogenases and uncoupling protein). Indeed, these alternative proteins are recognized as major stress response signals by acting as mitochondrial retrograde response (Gray et al. 2004, Dojcinovic et al. 2005). AOX is the best model to study retrograde signaling in plant mitochondria mainly due to its role against ROS generation resulting from both abiotic and biotic stress (Vanlerberghe 2013). Compelling evidence has demonstrated that AOX1a is regulated by $\mathrm{ABI} 4$ via retrograde response and that this involves the cyclin-dependent kinase E1 (CDKE1) as cascade signaling (Ho et al. 2008, Giraud et al. 2009, Blanco et al. 2014). Furthermore, both ABI4 and CDKE1 are responsive to $A B A$ and sugar signaling regulating energy status under stress response (Arroyo et al. 2003, Acevedo-Hernández et al. 2005, Blanco et al. 2014). Therefore, it seems reasonable to assume following carbohydrate starvation that mitochondrial metabolism triggers a proteome response and that low energy status is most likely mediated by AOX and other IMM protein.

Although it is recognized that mitochondria employ different alternative respiratory pathways under stress conditions, the regulation of proteins involved in stress-specific responses, like carbohydrate starvation, remains unclear. For this purpose, we further investigated the behavior of the mitochondrial respiratory complexes under carbohydrate starvation and how different amino acids affect the mETC (Figs 5 and 6). First, we have observed a reduction of complex $\mathrm{I}$ and alternative $\mathrm{NADH}$ dehydrogenases activities in absence of sucrose
(Fig. 5), and based on complex I-rotenone sensitive assay notably this reduction was likely associated to complex I. Carbohydrate starvation has already been shown decreased $\mathrm{NADH} / \mathrm{NAD}^{+}$ratio which might explain, at least partially, a reduction of complex I activity.

In addition, we evaluated whether this reduction in the activity of the complex I was associated with the reduction in the amount of protein. To this end, we focused on the $51 \mathrm{kDa}$ subunit of the complex I by immunoblotting assay and observed no changes in the amount of protein. The reasons for this may be twofold: (1) the $51 \mathrm{kDa}$ subunit might not reflect per se structural changes on complex I because this complex is comprised of more than 50 subunits in plants and, thus it is likely that investigation of other subunits of this complex are still required and/or (2) the $51 \mathrm{kDa}$ subunit as well as other mitochondrial enzymes are associated with post-translational modification (PTM) which are usually required in response to stress situations such as carbohydrate starvation. In fact, the $51 \mathrm{kDa}$ subunit was shown be a PTM target by phosphorylation (Haezlewwod et al. 2004, Braun et al. 2014). Moreover, reductions in the $\mathrm{NADH} / \mathrm{NAD}^{+}$ratio and carbon starvation in mice (Mus muscullus) were described to induce PTM mainly by deacetylation mediated by sirtuins (Ahn et al. 2008). It has also been recently demonstrated that in Arabidopsis PTM by acetylation are mediated by sirtuins (Finkemeier et al. 2011, König et al. 2014). In addition, components of the OXPHOS system have been suggested as potential targets of sirtuins and importantly among these targets it was shown that complex I is strongly affected (Finkemeier et al. 2011). However, proteome profiling revealed reductions in most of carbonic anhydrase subunits in -Suc and +BCAA conditions which also suggest lower complex I abundance (Table S3). Carbonic anhydrase is fundamental for complex I assembly (Braun et al. 2014) and its lack in a double mutant deficient in carbonic anhydrases 1 and 2 (ca1ca2) resulted in a dysfunction of complex I leading to enhanced electron entry to mECT through amino acids catabolism (Hildebrandt et al. 2015, Fromm et al. 2016). Our results also demonstrated that complex II activity is not affected in the presence of BCAA as the only carbon source (Fig. 3). Collectively, this result coupled with the activity of both IVDH, complexes I and II indicate that BCAA is able to promote alterations in the plant OXPHOS system which is clearly associated with the fact that the electrons donated by those amino acids go into the mETC by the ETF/ETFQO system directly to ubiquinone, thereby skipping complexes I and II, given that the catabolic products of BCAA degradation feed electrons directly into the TCA cycle. It is worth to mention, however, that energy yield following complete oxidation of BCAAs and lysine is particularly high 
(Hildebrandt et al. 2015) and that only a minor part of this energy production is directly associated with electrons transferred via the ETF/ETFQO pathway. Thus, despite the activation of this pathway following carbohydrate starvation, the maintenance of the activity of other complexes is also in line with our suggestions of a key role for those amino acids as an alternative source of electrons. On the other hand, it should be mentioned that lysine catabolism generates electrons that are transferred to the respiratory chain through to the ubiquinol pool through an ETF/ETFQO system, or, it might enter in the TCA cycle directly after being converted into 2-oxoglutarate and direct electron supply to the ubiquinone pool of the mETC through complex II (Araújo et al. 2011). Therefore, the occurrence of this pathway could explain, at least in part, the changes observed in the activity of the OXPHOS complexes of when lysine was used following carbohydrate starvation conditions (Fig. 5).

In summary, we have presented compelling evidence that following carbohydrate starvation changes in the OXPHOS system in Arabidopsis mitochondria and in the mitochondrial proteome are evident and that the responses are variable according the amino acid that is used as a carbon source during such stress conditions. Interestingly, BCAA supplementation is able to differentially affect $\mathrm{mETC}$ complexes although a similar response for lysine was observed for all complexes. Our results also demonstrated that there is a positive correlation between IVDH activity and protein amount detected by immunoblotting. Collectively, these data provide evidences concerning OXPHOS system regulation where ETF/ETFQO is of pivotal importance during plant stress responses.

\section{Materials and methods}

\section{Establishment of $A$. thaliana cell suspensions and induction of carbohydrate starvation stress}

All experiments were carried out using $A$. thaliana of the Columbia ecotype (Col-0). Establishment of an $A$. thaliana cell culture was carried out as described by May and Leaver (1993). Briefly, A. thaliana seeds were surface sterilized and imbibed for 2 days at $4^{\circ} \mathrm{C}$ in the dark on $0.8 \%(\mathrm{w} / \mathrm{v})$ agar plates containing half-strength Murashige and Skoog (MS) media ( $\mathrm{pH}$ 5.7). Seeds were subsequently germinated under long-day conditions (16 h light $/ 8 \mathrm{~h}$ dark) at $100 \mu \mathrm{mol} \mathrm{m}{ }^{-2} \mathrm{~s}^{-1}$.

After 10 days, seedlings were cut into small pieces $\left(3 \mathrm{~cm}^{2}\right)$ and laid out on plates with B5 medium $[0.316 \%$ $(\mathrm{w} / \mathrm{v}) \mathrm{B} 5,0.75 \mathrm{~g}(\mathrm{w} / \mathrm{v})$ agar, 3\% sucrose $(\mathrm{w} / \mathrm{v}), 0.0001 \%$ $(\mathrm{w} / \mathrm{v})$ 2,4-dichloropenoxyacetic acid and $0.00001 \%$ kinetin $(\mathrm{w} / \mathrm{v}), \mathrm{pH} 5.7 / \mathrm{KOH}]$ for callus induction. The
B5 plates were stored in darkness for 3 weeks. Afterwards, callus was transferred into liquid B5 medium (B5 medium without agarose) for establishing a cell suspension culture. Cells were cultivated in $500-\mathrm{ml}$ flasks containing $100 \mathrm{ml}$ of medium at $25^{\circ} \mathrm{C}$ and $90 \mathrm{rpm}$ in the dark. Cells were transferred into fresh medium all 7 days. The starting material for mitochondrial isolations (see below) was approximately $40 \mathrm{~g}$ cells.

Carbohydrate starvation was induced by removing sucrose from the suspension cell medium. For the experiments, defined amounts of sucrose or amino acids were added. Specifically, cell cultivation took place at the following four conditions: (1) control (3\% sucrose), (2) no sucrose, (3) BCAA [without sucrose plus $10 \mathrm{mM}$ BCAA (each isoleucine, leucine and valine, respectively)] and (4) lysine (without sucrose plus $30 \mathrm{mM}$ lysine) for $24 \mathrm{~h}$. This concentration is very close to the pool of free amino acid found in plants after stress (Urano et al. 2009, Hildebrandt et al. 2015, Pires et al. 2016). Additionally, controls with the addition of free amino acid have been performed initially but with no significant changes in cellular and physiological parameters. For this reason, we have further eliminated these treatments from our manuscript.

\section{Growth experiments}

Starting point of growth experiments was the transfer of $1.5 \mathrm{~g}$ of $A$. thaliana Col-0 cells into fresh suspension cell medium. The five cultivation conditions were described as above. Weight increase was determined after $24 \mathrm{~h}$.

\section{Respiration measurements}

Dark respiration of the cell cultures was measured in an oxygen electrode following a protocol detailed previously (Geigenberger and Stitt 2000). Measurements took place in a $2 \mathrm{ml}$ reaction vessel for about $5 \mathrm{~min}$ and were based on $30 \mathrm{mg}$ cells. Oxygen depletion in the buffer was kept to less than $20 \%$ of the initial value. Potassium cyanide $(1 \mathrm{mM} \mathrm{KCN})$ was used for cytochrome $c$ oxidase inhibition and salicylhydroxamic acid $(750 \mu M$ SHAM) for inhibition of AOX.

\section{Cell viability}

Fluorescein-diacetate staining was carried out as described by Widholm (1972) and visualized using the Axio Scope A1 Zeiss microscope. Image analysis was carried out using the Axiovision scope A1 software. Analyses were based on the evaluation scheme for cell clusters as described by Winkelmann et al. (1998), which is based on four viability classes: I 
(fully viable); II (2/3 viable); III (1/3 viable); IV (dead), and the viabilities were calculated by the following equation: Viability $(\%)=100 \times\left[\left(n_{1} \times 1\right)+\left(n_{\| I} \times 2 / 3\right)+\right.$ $\left.\left(n_{\text {III }} \times 1 / 3\right)+\left(n_{I V} \times 0\right) / n_{\text {total }}\right]$.

\section{Isolation of mitochondria by Percoll gradient ultracentrifugation}

Preparations of mitochondria were performed according to Schertl et al. (2014). In brief, Arabidopsis cells were disrupted in a Waring blender in buffer containing $450 \mathrm{mM}$ sucrose, $15 \mathrm{mM}$ 3-(N-morpholino) propanesulfonic acid (MOPS), $1.5 \mathrm{mM}$, EGTA, 0.6 (w/v) polyvinylpyrrolidone (PVP-40), $0.2 \%$ bovine serum albumin (BSA), 0.2\% phenylmethylsulfonyl fluoride (PMSF), pH $7.4(\mathrm{KOH})$. To remove cell debris the suspensions were centrifuged twice for $5 \mathrm{~min}$ at $2700 \mathrm{~g}$ and once for $10 \mathrm{~min}$ at $8300 \mathrm{~g}$. Afterwards, the supernatants containing the mitochondrial fractions were transferred to a new tube for a high-speed centrifugation at $17000 \mathrm{~g}$ for $10 \mathrm{~min}$. The mitochondrial pellets were resuspended in $3 \mathrm{ml}$ of washing buffer containing $300 \mathrm{mM}$ sucrose, $10 \mathrm{mM}$ MOPS, $1 \mathrm{mM}$ EGTA, $0.2 \mathrm{mM}$ PMSF, pH 7.2 $(\mathrm{KOH})$. Next, the mitochondrial suspensions were carefully placed on Percoll gradients and centrifuged for $90 \mathrm{~min}$ at $70000 \mathrm{~g}$ using an ultracentrifuge. After centrifugation, the bands representing the mitochondria were transferred into new tubes using a Pasteur pipette. The mitochondrial fractions were washed three times in resuspension buffer containing $400 \mathrm{mM}$ mannitol, $10 \mathrm{mM}$ Tricine, $1 \mathrm{mM}$ EGTA, $0.2 \mathrm{mM}$ PMSF, pH 7.2 $(\mathrm{KOH})$ at by centrifugation at $14300 \mathrm{~g}$ for $10 \mathrm{~min}$. All steps were carried out at $4^{\circ} \mathrm{C}$. Isolated mitochondria were aliquoted and stored at $-80^{\circ} \mathrm{C}$.

\section{Measurement of mitochondrial enzyme activity}

All enzyme assays were carried out in final volumes of $300 \mu \mathrm{l}$ at $25^{\circ} \mathrm{C}$ using an Epoch Microplate Spectrophotometer (Biotech, Winooski, VT). Mitochondrial suspensions stored at $-80^{\circ} \mathrm{C}$ were used to quantify the mitochondrial enzyme activities. First, mitochondrial suspensions were thawed and then centrifuged for $10 \mathrm{~min}$ at $25000 \mathrm{~g}$. The supernatants (soluble fractions) were recovered in a new microtube. The pellets were suspended in resuspension buffer (membrane fraction) and protein quantifications were carried out for both the soluble and the membrane fractions using the Bradford method (Bradford 1976).

IVDH activity was monitored by following the reduction of dichlorophenolindophenol (DCIP) $\left(E=19.1 \mathrm{mM}^{-1} \mathrm{~cm}^{-1}\right)$ at $600 \mathrm{~nm}$. The reaction mixture contained $250 \mathrm{mM}$ Tris- $\mathrm{HCl} \mathrm{pH} 7.5,1 \mathrm{mM} \mathrm{KCN}$, $166 \mu M$ FAD, $0.06 \mathrm{mM}$ DCIP, $1 \mathrm{mM}$ PMS and $20 \mu \mathrm{g}$ of soluble mitochondrial proteins. The reaction was started by adding $67 \mu \mathrm{M}$ Acyl-CoA.

To determine OXPHOS system activities we used membrane fractions to allow protein enrichment. Complex I and alternative NADH dehydrogenase activities were measured according to Birch-Machin et al. (1994). Mitochondrial proteins $(5 \mu \mathrm{g})$ were suspended in a buffer containing $0.25 \mathrm{mM}$ Tris- $\mathrm{HCl} \mathrm{pH} 7.5,2 \mathrm{mM}$ $\mathrm{KCN}, 2 \mu \mathrm{g} \mathrm{ml}^{-1}$ antimycin A, $0.2 \mathrm{mM} \mathrm{NADH}, 200 \mu \mathrm{M}$ ubiquinone (oxidized). Complex I activity was measured by following the decrease in absorbance due to the oxidation of $\mathrm{NADH}$ at $340 \mathrm{~nm}\left(E=6.2 \mathrm{mM}^{-1} \mathrm{~cm}^{-1}\right)$. The specific activity of complex I was measured before the addition of $2 \mu \mathrm{g} \mathrm{ml}^{-1}$ rotenone.

Complex II was measured according to Birch-Machin et al. (1994). For this assay, $15 \mu \mathrm{g}$ of membrane protein was suspended in a buffer including $50 \mathrm{mM}$ Tris- $\mathrm{HCl}$ $\mathrm{pH} 7.4,5 \mathrm{mM} \mathrm{MgCl} 2,20 \mathrm{mM}$ succinate, $0.3 \mathrm{mM}$ ATP, $0.5 \mathrm{mM}$ SHAM, $100 \mu \mathrm{M}$ decylubiquinone (oxidized) and $2 \mathrm{mM}$ KCN. The reaction was started with $50 \mu \mathrm{M}$ DCIP and the activity of complex II was monitored by the reduction of DCIP $\left(E=19.1 \mathrm{mM}^{-1} \mathrm{~cm}^{-1}\right)$ at $600 \mathrm{~nm}$.

Complex III activity was measured according Birch-Machin et al. (1994) and the preparation of decylubiquinol according to Lang and Packer (1987). The assay was carried out with $2 \mu \mathrm{g}$ of protein from mitochondrial membrane fraction and took place in a buffer including $50 \mathrm{mM}$ Tris-HCL pH 7.4, $5 \mathrm{mM} \mathrm{MgCl}_{2}, 2.5 \mathrm{mg} \mathrm{ml}^{-1} \mathrm{BSA}$, $2 \mathrm{mM} \mathrm{KCN}, 15 \mu \mathrm{M}$ oxidized cytochrome c, $0.005 \mu \mathrm{g} \mu \mathrm{I}^{-1}$ Rotenone, $35 \mu \mathrm{M}$ decylubiquinol. Enzyme activity was followed at $550 \mathrm{~nm}$ for $10 \mathrm{~min}$.

Complex IV activity was measured according to Birch-Machin et al. (1994). The assay mixture contained $50 \mathrm{mM}$ Tris- $\mathrm{HCl} \mathrm{pH} 7.4,15 \mu \mathrm{M}$ cytochrome $\mathrm{c}$ (reduced with sodium dithionite and afterwards gel filtrated using PD-10 Desalting Columns; GE Healthcare Europe $\mathrm{GmbH}$, Freiburg, Germany). Protein ( $1 \mu \mathrm{g}$ of membrane proteins) was used to start the reaction and oxidation of cytochrome $c\left(E=19 \mathrm{mM}^{-1} \mathrm{~cm}^{-1}\right)$ was monitored at $550 \mathrm{~nm}$.

\section{SDS-PAGE}

One-dimensional SDS-PAGE was carried out as outlined by Laemmli (1970). Mitochondrial aliquots kept at $-80^{\circ} \mathrm{C}$ were used as starting material. Before gel loading, mitochondrial aliquots were centrifuged for $10 \mathrm{~min}$ at $25000 \mathrm{~g}$ at $4^{\circ} \mathrm{C}$. Afterwards, soluble and membrane fractions were transferred into new tubes. Mitochondrial proteins were quantified using a microplate assay using Pierce Coomassie (Bradford) protein assay (Pierce Biotechnology, Rockford, IL). Samples $(10 \mu \mathrm{g})$ were mixed with loading buffer containing 
$8 \%(\mathrm{w} / \mathrm{v})$ SDS, $250 \mathrm{mM}$ Tris-HCl, $\mathrm{pH} 6.8,40 \%(\mathrm{v} / \mathrm{v})$ glycerol, $0.002 \%(\mathrm{v} / \mathrm{v})$ bromophenol blue, $2 \%$ mercaptoethanol and $0.05 \%(\mathrm{v} / \mathrm{v})$ dithiothreitol (DTT) and then incubated for $5 \mathrm{~min}$ at $70^{\circ} \mathrm{C}$ before application onto pre-cast $(8 \times 8 \mathrm{~cm})$ Tris-glycine SDS gels (Life technology, Carslbad, CA). The running conditions were as follows: $125 \mathrm{~V}, 30 \mathrm{~mA}$, running time about $2 \mathrm{~h}$ in glycine buffer [0.248 M Tris; $1.92 M$ Glycine; 1\% (w/v) SDS; pH not adjusted].

\section{Immunoblotting}

Proteins separated in polyacrylamide gels were transferred onto nitrocellulose membranes using a Semi-Dry Transfer system (Bio-Rad, Hercules, CA). The transfer of proteins was carried out according to Schertl et al. (2014) at $12 \mathrm{~V}$ (max) and $400 \mathrm{~mA}$ for about $90 \mathrm{~min}$ using transfer buffer $(25 \mathrm{mM}$ Tris, $192 \mathrm{mM}$ glycine, $1.3 \mathrm{mM}$ SDS, 20\% Methanol). The blots were rinsed several times in TTBS buffer [0.1 M Tris- $\mathrm{HCl}$ pH 7.5, $0.155 \mathrm{M}$ $\mathrm{NaCl}$ and $0.01 \%(\mathrm{v} / \mathrm{v})$ Tween 20 ] to remove all methanol and then incubated with 1:800 primary antibody diluted in TTBS buffer for about $16 \mathrm{~h}$ at room temperature. Antibodies for OXPHOS subunits are described in Peters et al. (2012) which are the $51 \mathrm{kDa}$ subunit (complex I), SDH1-1 (complex II), alpha-MPP (complex III), COX2 (complex IV) and the ß-subunit (complex V). The IVDH antibody was described by Gu et al. (2010). After antibody incubation, membranes were incubated for $2 \mathrm{~h}$ with a 1:5000 diluted secondary antibody directly coupled to horseradish peroxidase. All blots were developed using a homebrew protocol luminol chemical-based as following described [Tris $100 \mathrm{mM}$ pH 8.5; p-cumaric acid $(15 \% \mathrm{v} / \mathrm{v})$; luminol $\left.(44 \% \mathrm{v} / \mathrm{v}) ; \mathrm{H}_{2} \mathrm{O}_{2}(15 \% \mathrm{v} / \mathrm{v})\right]$ the $\mathrm{ECL}$ prime chemiluminescence detection kit from GE Healthcare (Munich, Germany).

\section{Protein separation and label free quantitative shotgun mass spectrometry}

Sample preparation and liquid chromatography coupled tandem mass spectrometry (LC-MS/MS) were performed as described in Fromm et al. (2016). In brief, mitochondrial proteins of three to four biological replicates were extracted and concentrated on top of a $14 \%(\mathrm{w} / \mathrm{v})$ polyacrylamide separation gel. After excision of the gel band, it was cut into cubes of approximately $1.5 \mathrm{~mm}$ and samples were first reduced by DTT followed by alkylation with iodoacetamide, tryptic digestion and peptide extraction. For MS analysis, samples were reconstituted in $20 \mu \mathrm{l}$ of a solution containing $2 \%(\mathrm{v} / \mathrm{v})$ acetonitrile $(\mathrm{ACN})$ and $0.1 \%(\mathrm{v} / \mathrm{v})$ formic acid (FA). Tandem mass spectrometry (MS/MS) analysis was performed using a Q-Exactive (Thermo Fisher Scientific, Dreieich, Germany) mass spectrometer coupled to an Ultimate 3000 (Thermo Fisher Scientific) UPLC. Peptides were eluted from the $\mathrm{C} 18$ reverse phase column during a $60 \mathrm{~min} \mathrm{ACN}$ non-linear gradient $(2-30 \%)$. UPLC and MS settings were carried out exactly as described previously (Fromm et al. 2016). MS/MS spectra were queried against an in-house Arabidopsis protein database also containing frequent contaminations and RNA-editing sites using the Andromeda search engine as part of the MaxQuant (Cox and Mann 2008) software package. Frequent contaminants (BSA, keratin, trypsin) were removed from the final output lists. MaxQuant analysis was performed using version 1.5.2.8. with the same parameter settings as described previously (Fromm et al. 2016). Allowed protein modifications include carbamidomethylation of cysteine residues (static), acetylation of the $\mathrm{N}$-terminus and oxidation of methionines (both variable). The number of missed cleavages was set to two. Label-free quantification (LFQ) intensities from the corresponding MaxQuant file were uploaded into the Perseus software and quantitation matrix was built based on log2-transformed LFQ intensity values. Only proteins with a $P$ value $<0.05$ were considered statistically significant. Functional categorization enrichment was performed using PageMan package into MapMan software (Usadel et al. 2009). The analysis was performed using the Wilcoxon's test through total protein identified in LC-MS/MS assay. Further information is additionally available in Table S5.

\section{Statistical analysis}

Data were evaluated by variance analysis and tested for significance differences $(P<0.05)$ using Student's $t$-tests. All statistical analyses were performed using algorithms embedded into Microsoft Excel ${ }^{\circledR}$.

\section{Author contributions}

T. H., A. N.-N., H.-P. B. and W. L. A. designed the research; J. H. F. C., C. G. S. Q. and D. S. B. performed the research; P. S., D. S. B. and H. E. contributed new reagents/analytic tools; J. H. F. C., C. G. S. Q., H. E., T. H., A. N.-N., H.-P. B. and W. L. A. analyzed the data; J. H. F. C., C. G. S. Q., H.-P. B. and W. L. A. wrote the article with input from all the others. The authors declare that there is no conflict of interest.

Acknowledgements - We would like to thank Prof. Dr Traud Winkelmann (Leibniz Universität Hannover) for kindly providing her laboratory physical space and equipment for performing part of this research. Furthermore, we gratefully acknowledge Christa Ruppelt, Dagmar Lewejohann, and 
Marianne Langer for excellent technical assistance. This work was supported by supported by the binational science funding program 'PROBRAL' of the Deutsche Akademische Austauschdienst (DAAD; funds provided by the Bundesministerium für Bildung und Forschung - BMBF) and the Coordenação de Aperfeiçoamento de Pessoal de Nível Superior (CAPES), Refs: Project-ID PROBRAL $\# 423 / 14$. Research fellowships granted by the Foundation for Research Assistance of the Minas Gerais State (FAPEMIG-Brazil grant \# BDP-00018-16) to J. H. F. C. and the National Council for Scientific and Technological Development (CNPq-Brazil) to A. N. N. and W. L. A. are gratefully acknowledged.

\section{References}

Acevedo-Hernádez GJ, León P, Herrera-Estrella LR (2005) Sugar and ABA responsiveness of a minimal RBCS light-responsive unit is mediated by direct binding of ABI4. Plant J 43: 506-519

Ahn BH, Kim HD, Song S, Lee IH, Vassilopoulos A, Deng CX, Finkel T (2008) A role for the mitochondrial deacetylase Sirt3 in regulation energy homeostasis. Proc Natl Acad Sci U S A 105: 14447-14452

Araújo WL, Ishizaki K, Nunes-Nesi A, Larson TR, Tohge T, Krahnert I, Witt S, Obata T, Schauer N, Graham IA, Leaver CJ, Fernie AR (2010) Identification of the 2-hydroxyglutarate and isovaleryl-CoA dehydrogenases as alternative electron donors linking lysine catabolism to the electron transport chain of Arabidopsis mitochondria. Trends Plant Sci 22: 1549-1563

Araújo WL, Tohge T, Ishizaki K, Leaver CJ, Fernie AR (2011) Protein degradation - an alternative respiratory substrate for stressed plants. Trends Plant Sci 16: 489-498

Araujo WL, Nunes-Nesi A, Nikoloski Z, Sweetlove LJ, Fernie AR (2012) Metabolic control and regulation of the tricarboxylic acid cycle in photosynthetic and heterotrophic plant tissues. Plant Cell Environ 35: 1-21

Arroyo A, Bossi F, Finkelstein RR, León P (2003) Three genes that affect sugar sensing (abscisic acid insensitive 4, abscisic acid insensitive 5, and constitutive triple response 1) are differentially regulated by glucose in Arabidopsis. Plant Physiol 133: 231-242

Beckmann JD, Frerman FE (1985) Reaction of electron-transfer flavoprotein with electron transfer flavoprotein-ubiquinone oxidoreductase. Biochemistry 24: 3922-3925

Birch-Machin MA, Briggs HL, Saborido AA, Bindoff LA, Turnbull DM (1994) An evaluation of the measurement of the activities of complexes I-IV in the respiratory chain of human skeletal muscle mitochondria. Biochem Med Metab Biol 51: 35-42

Blanco NE, Guinea-Díaz M, Whelan J, Strand A (2014) Interaction between plastid and mitochondrial retrograde signalling pathways during changes to plastid redox status. Philos Trans R Soc Lond B Biol Sci 369: 20130231

Bradford MM (1976) A rapid and sensitive method for the quantitation of microgram quantities of protein utilizing the principle of protein-dye binding. Anal Biochem 72: 248-254

Braun HP, Binder S, Brennicke A, Eubel H, Fernie AR, Finkemeier I, Klodman J, König AC, Kühn K, Meyer E, Obata T, Schwarzländer M, Takenaka M, Zehrmann A (2014) The life of plant mitochondrial complex I. Mitochondrion 19: 295-313

Brouwer KS, van Valen T, Day DA, Lambers H (1986) Hydroxamate-stimulated $\mathrm{O}_{2}$ uptake in roots of Pisum sativum and Zea mays mediated by a peroxidase: its consequences for respiration measurements. Plant Physiol 82: 236-240

Buchanan-Wollaston $V$, Page T, Harrison E, Breeze E, Lim PO, Nam HG, Lin JF, SH W, Swidzinski J, Ishizaki K, Leaver CJ (2005) Comparative transcriptome analysis reveals significant differences in gene expression and signalling pathways between developmental and dark/starvation-induced senescence in Arabidopsis. Plant J 42: $567-585$

Cavalcanti JHF, Esteves-Ferreira AA, Quinhones CGS, Pereira-Lima IA, Nunes-Nesi A, Fernie AR, Araújo WL (2014) Evolution and functional implications of the tricarboxylic acid cycle as revealed by phylogenetic analysis. Genome Bio Evol 6: 2830-2848

Chase CD (2007) Cytoplasmic male sterility: a window to the world of plant mitochondrial nuclear interactions. Trends Genet 23: 81-90

Contento AL, Kim SJ, Bassham DC (2004) Transcriptome profiling of the response of Arabidopsis suspension culture cells to Suc starvation. Plant Physiol 135: 2330-2347

Cox J, Mann M (2008) MaxQuant enables high peptide identification rates, individualized p.P.B.-range mass accuracies and proteome-wide protein quantification. Nat Biotechnol 26: 1367-1372

de Visser R, Blacquière T (1984) Inhibition and stimulation of root respiration in Pisum and Plantago by hydroxamate. Its consequences for the assessment of alternative path activity. Plant Physiol 75: 813-817

Dojcinovic D, Krosting J, Harris AJ, Wagner DJ, Rhoads DM (2005) Identification of a region of the Arabidopsis AtAOX1a promoter necessary for mitochondrial retrograde regulation of expression. Plant Mol Biol 58: $159-175$

Engqvist M, Drincovich MF, Fluegge U-I, Maurino VG (2009) Two D-2-hydroxy- acid dehydrogenases in Arabidopsis thaliana with catalytic capacities to participate in the last reactions of the methyl glyoxal and beta-oxidation pathways. J Biol Chem 84: 25026-25037 
Engqvist M, Kuhn A, Wienstroer J, Weber K, Jansen EEW, Jakobs C, Weber APM, Maurino VG (2011) Plant D-2-Hydroxyglutarate dehydrogenase participates in the catabolism of lysine especially during senescence. J Biol Chem 286: 11382-11390

Fernie AR, Carrari F, Sweetlove LJ (2004) Respiratory metabolism: glycolysis, the TCA cycle and mitochondrial electron transport. Curr Opin Plant Biol 7: 254-261

Finkemeier I, Laxa M, Miguet L, Howden AJ, Sweetlove LJ (2011) Proteins of diverse function and subcellular location are lysine acetylated in Arabidopsis. Plant Physiol 155: 1179-1790

Fromm S, Senkler J, Eubel H, Peterhänsel C, Braun H-P (2016) Life without complex I: proteome analyses of an Arabidopsis mutant lacking the mitochondrial NADH dehydrogenase complex. J Exp Bot 67: 3079-3093

Galili G (2011) The aspartate-family pathway of plants linking production of essential amino acids with energy and stress regulation. Plant Signal Behav 6: 192-195

Galili G, Avin-Wittenberg T, Angelovici R, Fernie AR (2014) The role of photosynthesis and amino acid metabolism in the energy status during seed development. Front Plant Sci 5: 447

Geigenberg P, Fernie AR, Gibon Y, Christ M, Stitt M (2000) Metabolic activity decreases as an adaptive response to low internal oxygen in growing tomato tubers. Biol Chem 381: 723-740

Geigenberger P, Stitt M (2000) Diurnal changes in sucrose, nucleotides, starch synthesis and AGPS transcript in growing potato tubers that are suppressed by decreased expression of sucrose phosphate synthase. Plant J 236: 795

Giraud E, Van Aken O, Ho LH, Whelan J (2009) The transcription factor $\mathrm{ABI} 4$ is a regulator of mitochondrial retrograde expression of ALTERNATIVE OXIDASE1a. Plant Physiol 150: 1286-1296

Gray GR, Maxwell DP, Villarimo AR, Mclntosh L (2004) Mitochondria/nuclear signaling of alternative oxidase gene expression occurs through distinct pathways involving organic acids and reactive oxygen species. Plant Cell Rep 23: 497-503

Gu L, Jones AD, Last RL (2010) Broad connections in the Arabidopsis seed metabolic network revealed by metabolite profiling of an amino acid catabolism mutant. Plant J 614: 579-590

Heazlewood JL, Tonti-Filippini JS, Gout AM, Day DA, Whelan J, Millar AH (2004) Experimental analysis of the Arabidopsis mitochondrial proteome highlights signaling and regulatory components provides assessement of target prediction programs and indicates plant-specific mitochondrial proteins. Plant Cell 16: 241-256

Hildebrandt TM, Nunes-Nesi A, Araujo WL, Braun HP (2015) Amino acid catabolism in plants. Mol Plant 8: $1563-1579$
Ho LH, Giraud E, Uggalla V, Lister R, Clifton R, Glen A, Thirkettle-Watts D, Van Aken O, Whelan J (2008) Identification of regulatory pathways controlling gene expression of stress-responsive mitochondrial proteins in Arabidopsis. Plant Physiol 147: 1858-1873

Huang S, Shingaki-Wells RN, Taylor NL, Millar AH (2013) The rice mitochondria proteome and its response during development and to the environment. Front Plant Sci 4: 16

Ishizaki K, Larson TR, Schauer N, Fernie AR, Graham IA, Leaver CJ (2005) The critical role of Arabidopsis electron-transfer flavoprotein: ubiquinone oxidoreductase during dark induced starvation. Plant Cell 17: 2587-2600

Ishizaki K, Schauer N, Larson TR, Graham IA, Fernie AR, Leaver CJ (2006) The mitochondrial electron transfer flavoprotein complex is essential for survival of Arabidopsis in extended darkness. Plant J 47: 751-760

Journet EP, Bligny R, Douce R (1986) Biochemical changes during sucrose deprivation in higher plant cells. J Biol Chem 261: 3193-3199

Kim SW, Koo BC, Kim J, Liu JR (2007) Metabolic discrimination of sucrose starvation from Arabidopsis cell suspension by $1 \mathrm{H}$ NMR based metabolomics. Biotechnol Bioprocess Eng 12: 653-661

Kirma M, Araújo WL, Fernie AR, Galili G (2012) The multifaceted role of aspartate-family amino acids in plant metabolism. J Exp Bot 63: 4995-5001

Kleessen S, Araújo WL, Fernie AR, Nikiloski Z (2012) Model-based confirmation of alternative substrates of mitochondrial electron transport chain. J Biol Chem 287: 11122-11131

Klodmann J, Senkler M, Rode C, Braun H-P (2011) Defining the protein complex proteome of plant mitochondria. Plant Physiol 157: 587-598

König AC, Hartl M, Pham PA, Laxa M, Boersema PJ, Orwat A, Kalitventseva I, Plöchinger M, Braun HP, Leister D, Mann M, Wachter A, Fernie AR, Finkemeier I (2014) The Arabidopsis class II sirtuin is a lysine deacetylase and interacts with mitochondrial energy metabolism. Plant Physiol 164: 1401-1414

Kravchik M, Bernstein N (2013) Effects of salinity on the transcriptome of growing maize leaf cells point at cell-age specificity in the involvement of the antioxidative response in cell growth restriction. BMC Genomics 14: 1

Krüßel L, Junemann J, Wirtz M, Birke H, Thornton JD, Browning LW, Poschet G, Hell R, Balk J, Braun HP, Hildebrandt TM (2014) The mitochondrial sulfur dioxygenase ethylmalonic encephalopathy protein 1 is required for amino acid catabolism during carbohydrate starvation and embryo development in Arabidopsis. Plant Physiol 165: 92-104

Kühn K, Yin G, Duncan O, Law SR, Kubiszewski-Jakubiak S, Kaur P, Mayer E, Wang Y, Small CCF, Giraud E, 
Narsai R, Whelan J (2015) Decreasing electron flux through the cytochrome and/or alternative respiratory pathways triggers common and distinct cellular responses dependent on growth conditions. Plant Physiol 167: 228-250

Kruger NJ, Masakapalli SK, Ratcliffe RG (2012) Strategies for investigating the plant metabolic network with steady-state metabolic flux analysis: lessons from an Arabidopsis cell culture and other systems. J Exp Bot 63: 2309-2323

Laemmli UK (1970) Cleavage of structural proteins during the assembly of the head of bacteriophage T4. Nature 227: 680-685

Lang JK, Packer L (1987) Quantitative determination of vitamin E and oxidized and reduced coenzyme Q by high-performance liquid chromatography with in-line ultraviolet and electrochemical detection. J Chromatogr 385: 109-117

Masakapalli SK, Bryant FM, Kruger NJ, Ratcliffe RG (2014) The metabolic flux phenotype of heterotrophic Arabidopsis cells reveals a flexible balance between the cytosolic and plastidic contributions to carbohydrate oxidation in response to phosphate limitation. Plant J 78: 964-977

May MJ, Leaver CJ (1993) Oxidative stimulation of glutathione synthesis in Arabidopsis thaliana suspension cultures. Plant Physiol 103: 621-627

Millar AH, Heazlewood JL, Kristensen BK, Braun HP, Møller IM (2005) The plant mitochondrial proteome. Trends Plant Sci 10: 36-43

Millar AH, Whelan J, Soole KL, Day DA (2011) Organization and regulation of mitochondrial respiration in plants. Annu Rev Plant Biol 62: 79-104

Mohsen AA, Vockley J (2015) Kinetic and spectral properties of isovaleryl-CoA dehydrogenase and interaction with ligands. Biochimie 108: 108-119

Nicolai M, Roncato MA, Canoy AS, Rouquie D, Sarda X, Freyssinet G, Robaglia C (2006) Large-scale analysis of mRNA translation states during sucrose starvation in Arabidopsis cells identifies cell proliferation and chromatin structure as targets of translational control. Plant Physiol 141: 663-673

Obata T, Matthes A, Koszior S, Lehmann M, Araújo WL, Bock R, Sweetlove LJ, Fernie AR (2011) Alteration of mitochondrial protein complexes in relation to metabolic regulation under short-term oxidative stress in Arabidopsis seedlings. Phytochemistry 72: 1081-1091

Paxton WC, Podestá FE (2006) The functional organization and control of plant respiration. Crit Rev Plant Sci 25: 159-198

Peng C, Uygun S, Shiu SH, Last RL (2015) The impact of the branched-chain ketoacid dehydrogenase complex on amino acid homeostasis in Arabidopsis. Plant Physiol 169: $1807-1820$
Peters K, Nießes PC, Späth B, Hölzle A, Binder S, Marchfelder A, Braun HP (2012) Complex I-complex II ratio strongly differs in various organs of Arabidopsis thaliana. Plant Mol Biol 79: 273-284

Pires MV, Pereira Junior AA, Medeiros DB, Daloso DM, Pham PA, Barros KA, Engqvist MKM, Florian A, Krahnert I, Maurino VG, Araújo WL, Fernie AR (2016) The influence of alternative pathways of respiration that utilize branched-chain amino acids following water shortage in Arabidopsis. Plant Cell Environ 39: 1304-1319

Reape TJ, Molony EM, McCabe PF (2008) Programmed cell death in plants: distinguishing between different modes. J Exp Bot 59: 435-444

Roby C, Martin JB, Bligny R, Douce R (1987) Biochemical changes during sucrose deprivation in higher plant cells phosphorus-31 nuclear magnetic resonance studies. J Biol Chem 262: 5000-5007

Schertl P, Cabassa C, Saadallah K, Bordenave M, Savoure A, Braun HP (2014) Biochemical characterization of proline dehydrogenase in Arabidopsis mitochondria. FEBS J 281: 2794-2804

Schertl P, Braun H-P (2014) Respiratory electron transfer pathways in plant mitochondria. Front Plant Sci 5163: 7

Urano K, Maruyama K, Ogata Y, Morishita Y, Takeda M, Sakurai N, Suzuki H, Saito K, Shibata D, Kobayashi M, Yamaguchi-Shinozaki K, Shinozaki K (2009)

Characterization of the ABA-regulated global responses to dehydration in Arabidopsis by metabolomics. Plant J 57: 1065-1078

Usadel B, Nagel A, Steinhauser D, Gibon Y, Bläsing OE, Redestig H, Sreenivasulu N, Krall L, Hannah MA, Poree F, Fernie AR, Stitt M (2006) PageMan: an interactive ontology tool to generate, display, and annotate overview graphs for profiling experiments. BMC Bioinformatics 7: 535-553

Usadel B, Poree F, Nagel A, Lohse M, Czedik-Eysenberg A, Stitt M (2009) A guide to using MapMan to visualize and compare omics data in plants: a case study in the crop species, Maize. Plant Cell Environ 32: 1211-1129

Vanlerberghe GC (2013) Alternative oxidase: a mitochondrial respiratory pathway to maintain metabolic and signaling homeostasis during abiotic and biotic stress in plants. Int J Mol Sci 14: 6805-6847

Weigelt $K$, Küster $H$, Radchuk R, Müller M, Weichert $H$, Fait A, Fernie AR, Saalbach I, Weber H (2008) Increasing amino acid supply in pea embryos reveals specific interactions of $\mathrm{N}$ and $\mathrm{C}$ metabolism, and highlights the importance of mitochondrial metabolism. Plant J 55: 909-926

Widholm JM (1972) The use of fluorescein diacetate and phenosaphranine for determining viability of cultured plant cell. Stain Technol 47: 189-194 
Winkelmann T, Hohe A, Schwenkel HG (1998)

Establishing embryogenic suspension cultures in Cyclamen persicum 'purple flamed'. Adv Hort Sci 12: 25-30

Yao N, Eisfelder BJ, Marvin J, Greenberg JT (2004) The mitochondrion-an organelle commonly involved in programmed cell death in Arabidopsis thaliana. Plant J 40: 596-610

Zhou G, Jiang W, Zhao Y, Ma G, Xin W, Yin J, Zhao B (2003) Sodium tanshinone IIA sulfonate mediates electron transfer reaction in rat heart mitochondria. Biochem Pharmacol 65: 51-57

Zhu X, Galili G (2004) Lysine metabolism is concurrently regulated by synthesis and catabolism in both reproductive and vegetative tissues. Plant Physiol 135: $129-136$

\section{Supporting Information}

Additional Supporting Information may be found in the online version of this article:

Table S1. List of all proteins identified by UPLC/MS/MS.

Table S2. List of upregulated proteins.

Table S3. List of downregulated proteins.

Table S4. Amino-acid catabolism related proteins.

Table S5. Number of protein cluster according functional category.

Fig. S1. PageMan analysis revealing over-representative functional categorization of identified proteins. 